
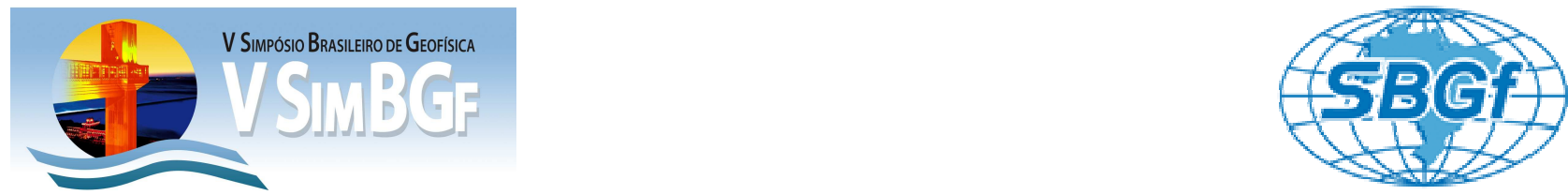

\title{
Caracterização geotécnica da seção marinha rasa empregando dados sísmicos - Vantagens e limitações da abordagem quantitativa
}

Jorge Fiori Fernandes Sobreira (*), PETROBRAS - E-mail: Fiori@petrobras.com.br

Marlos Lipski, PETROBRAS

Luciano Arêas Carvalho, Schlumberger

Fredy Alex Villaorduna Artola, Schlumberger

Copyright 2012, SBGf - Sociedade Brasileira de Geofísica

Este texto foi preparado para a apresentação no V Simpósio Brasileiro de Geofísica, Salvador, 27 a 29 de novembro de 2012. Seu conteúdo foi revisado pelo Comitê Técnico do V SimBGf, mas não necessariamente representa a opinião da SBGf ou de seus associados. É proibida a reprodução total ou parcial deste material para propósitos comerciais sem prévia autorização da SBG.

\section{Abstract}

Obtaining information embedded with geotechnical significance from rather indirect, however cheaper sources (as might just as well be the case of seismic data) could be useful in several instances, as in the case of the Oil Industry, where detailed knowledge of the nature and mechanical behavior of the shallow section is required whenever new infrastructure is to be installed offshore. This must be stated as valid provided the limitations of such an approach are previously acknowledged. One of these is the lack of known analytical relationships between the mostly used geotechnical parameters (such as undrained shear strength or Su) and the typical seismic attributes (such as compressional and shear velocity, here included the related elastic moduli, such as Lamés parameters). To overcome this, a quantitative approach was attempted, that allowed to establish empirically derived mathematical relationships between some of the main geotechnical parameters and some of the mostly used seismic elastic moduli. After an encouraging initial qualitative approach that suggested a direct relationship between those geotechnical and seismic quantities, such a quantitative approach was tested on two areas offshore Brazil and the results thus obtained demonstrated the applicability of the method, provided intrinsic limitations of such an approach are acknowledged.

\section{Introdução}

A obtenção de informação geotécnica direta para fins de Engenharia no ambiente offshore requer que sejam empreendidas campanhas dedicadas e geralmente de custo considerável. Além disso, o caráter da informação obtida é antes localizado, visto basear-se em furos rasos isolados, distribuídos de maneira esparsa, muitas vezes sem o conhecimento prévio das variações esperadas no âmbito regional. A obtenção deste mesmo tipo de informação, ainda que indiretamente, a partir de outras fontes, como os dados geofísicos (sísmicos, no caso) surge como uma alternativa potencial atrativa, na medida em que os mesmos estão geralmente disponíveis, demandados que são por outros segmentos da Indústria de Petróleo (o que tende a reduzir drasticamente os custos de obtenção da informação desejada), sem contar com o benefício de sua distribuição, geralmente regional e ampla, em oposição aos dados geotécnicos dedicados.

No caso da Sísmica, as ondas superficiais (ondas Scholte, no caso da interface água-sedimento, como o fundo do mar) são reconhecidas pela sua capacidade em fornecer informações relevantes da seção mais rasa, justamente onde reside o interesse geotécnico (isto é, até os primeiros 100-150 m abaixo do fundo do mar). No entanto, por questões relacionadas à sua origem, tais tipos de onda tendem a não estar representados nos registros sísmicos em águas profundas, que tem sido justamente o foco da atividade de E\&P no Brasil. Assim, outros modos de onda, com as ondas de corpo (compressionais e, desejavelmente, cisalhantes) devem ser considerados, na medida em que se busquem alternativas para se extrair indiretamente informação com significado geotécnico.

Em trabalhos anteriores, Sobreira et al. ( 2009, 2010a, 2010b, 2010c) demonstraram, numa abordagem eminentemente qualitativa, o relacionamento potencial direto entre parâmetros geotécnicos de emprego tradicional na Engenharia como o Su (undrained shear strength, que mede a resistência "não drenada" de solos ao cisalhamento) e os módulos elásticos derivados de grandezas sísmicas, em especial aqueles relacionados à onda cisalhante, como o módulo de cisalhamento $(\mu)$. Posteriormente, Sobreira et al. (2011) exemplificaram o emprego de uma abordagem mais quantitativa, visando à obtenção, de pseudoperfis geotécnicos (assim chamados por serem derivados de maneira indireta), em substituição aos perfis geotécnicos obtidos diretamente no campo. Em todos esses trabalhos, a investigação concentrou-se em duas áreas da Bacia de Campos, situada na Margem Continental Leste Brasileira, onde informação geotécnica dedicada estava disponível para fins de calibração e posterior comparação, a saber, os campos de Albacora e Marlim.

Este trabalho pretende discutir de maneira mais específica as vantagens e limitações do emprego desta última abordagem, de caráter quantitativo, ressaltando assim pontos com relação aos quais deve-se ter plena consciência antes de se aplicar a metodologia desenvolvida. 


\section{Metodologia e problema investigado}

Parâmetros geotécnicos de interesse para a Engenharia são obtidos a partir de furos geotécnicos rasos, em que se mede a resistência do solo à penetração de um dispositivo conhecido como CPT ou cone-penetrômetro. O parâmetro mais tipicamente empregado neste tipo de investigação é o chamado Su ou undrained shear strength, assim definido (equações 1 e 2):

$S u=\frac{\text { qnet }}{N_{\text {hit }}}$ (equação 1),

onde

$q$ net $=q t-\sigma_{w 0} \quad$ (equação 2),

sendo qnet a resistência "líquida" do cone, qt a resistência total do cone (já corrigida dos efeitos de geometria do cone e pressão de poro em excesso), Nkt é um fator específico para cada área, variável com as condições e propriedades do solo (como a plasticidade) e $\sigma_{\mathrm{v} 0}$ é o stress vertical referente à pressão total de sobrecarga em relação ao fundo do mar.

O parâmetro Su é normalmente expresso em unidades de pressão, como kPa. Para fins de uma correlação mais eficaz na comparação com parâmetros extraídos da Sísmica, privilegiaram-se aqueles com a mesma dimensão (de pressão) de Su, caso dos módulos elásticos (dinâmicos) - como o módulos de bulk ( $k$ ), Young $(E)$ e de cisalhamento $(\mu)$, derivados via inversão sísmica elástica (isto é, simultânea e/ ou conjunta) embora os parâmetros mais tradicionais, como Vp, Vs, densidade e as impedâncias acústica e cisalhante também tendam a apresentar uma correspondência positiva com aqueles parâmetros geotécnicos. O módulo de cisalhamento, por exemplo, é definido como

$\mu=V s^{2}$.rho (equação 3),

sendo Vs a velocidade cisalhante e rho a densidade.

A inversão sísmica elástica foi realizada em duas áreas da Bacia de Campos, a saber: Albacora (onde dados sísmicos multicomponentes, 4C, estavam disponíveis) e Marlim (onde dados sísmicos apenas de onda $P$, porém de alta resolução e com riqueza de afastamentos - útil para empreender inversão do tipo AVO - estavam disponíveis). Em ambas as áreas, furos geotécnicos, alguns incluindo perfis de velocidade cisalhante (Vs) bem como medidas de densidade obtidos in situ puderam também ser utilizados e foram essenciais para fins de calibração ou posterior comparação com os resultados obtidos.

A metodologia desenvolvida para a extração de parâmetros geotécnicos baseou-se em duas abordagens, uma qualitativa, inicial, e outra quantitativa, brevemente descritas a seguir.

\section{Abordagem qualitativa - principais resultados}

A etapa inicial do trabalho baseou-se na comparação essencialmente qualitativa dos dados geotécnicos diretos com diferentes parâmetros e módulos elásticos derivados por inversão sísmica elástica. Esta abordagem encontrase bem detalhada em trabalhos anteriores (Sobreira, 2009, 2010a, 2010b, 2010c). A melhor correlação foi considerada para aqueles módulos elásticos associados à onda cisalhante (que se relaciona, essencialmente, às características de rigidez do arcabouço, o que, em última análise, controla as propriedades de interesse para a Geotecnia), caso dos módulos de Young e de cisalhamento. Esta importância da incorporação da informação genuinamente elástica ao processo, conferindo especial robustez aos resultados, foi objeto de trabalho anterior (Sobreira et al., 2010c). Um exemplo daquela correlação pode ser observado na figura 1 (para o pé do talude da região do campo de Marlim), onde importante contraste geotécnico, conhecido, entre os sedimentos subaflorantes mais rígidos (e neste caso também menos jovens) do talude e sedimentos menos rígidos (e neste caso, também mais jovens) do sopé é atestado pelos valores diferenciados do modulo de cisalhamento. Ambos os sedimentos estão sotopostos a uma delgada cobertura sedimentar quaternária e inconsolidada ("drape"). Nesta mesma figura pode ser observado que os principais contrastes do perfil de Su de um furo geotécnico localizado nas proximidades tendem a ser qualitativamente reproduzidos pelos valores do módulo de cisalhamento.

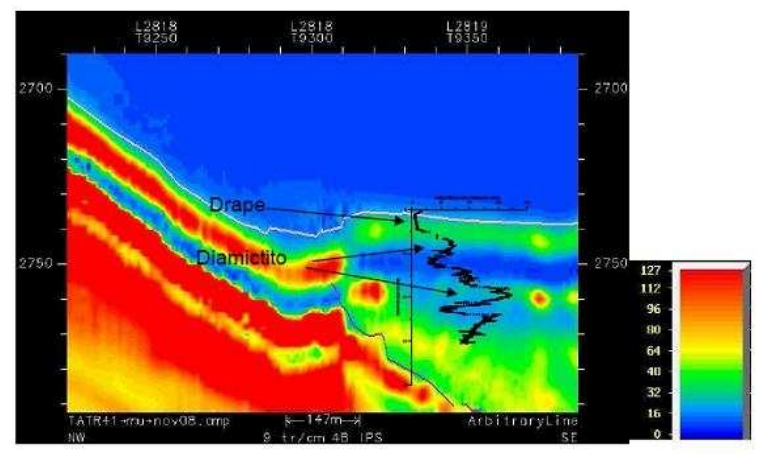

Figura 1 - Detalhe da seção do módulo de cisalhamento derivado de inversão sísmica elástica na região do pé do talude em Marlim, com perfil de $\mathrm{Su}$ (com profundidade - na vertical - variável de 0 a $30 \mathrm{~m}$, e magnitude na horizontal - variável de 0 a $160 \mathrm{kPa}$ ) de um furo geotécnico próximo, superposto para fins de comparação. A seção varia de 2.690 a 2.790 ms (tempo sísmico duplo) - na vertical - e tem cerca de $1 \mathrm{~km}$ de extensão horizontal. A escala de cores para o módulo de cisalhamento varia de 0 (azul escuro) a 127 (vermelho). 
Os resultados desta etapa qualitativa não só encorajaram a realização de outra, já com abordagem eminentemente quantitativa, como forneceram subsídios valiosos para a mesma.

\section{Abordagem quantitativa - principais resultados}

A etapa quantitativa do estudo, melhor detalhada em Sobreira et al., 2011, focou na busca, para cada área investigada, das relações matemáticas que melhor ajuste promoveram entre os principais parâmetros geotécnicos, como Su e qnet, e os módulos elásticos definidos na etapa anterior, a saber, módulos de bulk, de Young e de cisalhamento. Buscaram-se as relações que correspondessem aos melhores coeficientes de correlação, relações estas obtidas empiricamente (isto é, derivadas de crossplote entre cada par de parâmetros geotécnico-módulo elástico). O módulo de bulk ou incompressibilidade (relacionado apenas à onda compressional) foi incluído apenas para fins de comparação, pois já se era sabido, da etapa qualitativa anterior, da sua correlação mais fraca com os parâmetros geotécnicos em relação aos demais módulos elásticos.

A relação matemática escolhida em termos de melhor ajuste foi aquela do tipo "power law" (genericamente, da forma $\left.y=a . x^{b}\right)$, onde " $y$ " e " $x$ " são as variáveis a serem crossplotadas, e "a" e "b" são coeficientes, particulares para cada área considerada, o primeiro relacionado à inclinação da curva obtida e o segundo à sua concavidade. Um exemplo dos resultados obtidos nesta etapa é apresentado na figura 2 (curvas de melhor ajuste entre o módulo de cisalhamento e Su para Albacora). Como exemplo, a expressão matemática empiricamente derivada que forneceu o melhor ajuste (para o caso "global", vide legenda da figura) foi:

Sup $=2,0869 * 10^{5} * \mu^{1.1896}$,

onde Sup indica "Pseudo-Su" ou o Su previsto a partir da Sísmica (neste caso, a partir do módulo de cisalhamento).

A figura 3, por outro lado, ilustra uma pseudo-seção geotécnica em profundidade (de Su no caso) da área de Marlim (por "pseudo" aqui, entende-se aqueles parâmetros, perfis, seções ou volumes previstos a partir de dados sísmicos, segundo a metodologia desenvolvida e aplicada, e não diretamente obtidos no campo por testes geotécnicos). Já a figura 4 representa um detalhe desta figura, mostrando a comparação com furos geotécnicos reais. A obtenção de volumes ou seções de pseudo-parâmetros geotécnicos (como pseudo-Su, por exemplo) permite prever-se o comportamento geotécnico da área investigada, na medida em que pseudo-perfis geotécnicos possam ser gerados em pontos arbitrários daqueles volumes ou seções, possibilitando a comparação com perfis geotécnicos obtidos diretamente no campo ("blind test"). Uma seção deste tipo, que pode ser apresentada em tempo, ou ainda, ser convertida em profundidade, corresponde a um típico produto da etapa quantitativa do trabalho.

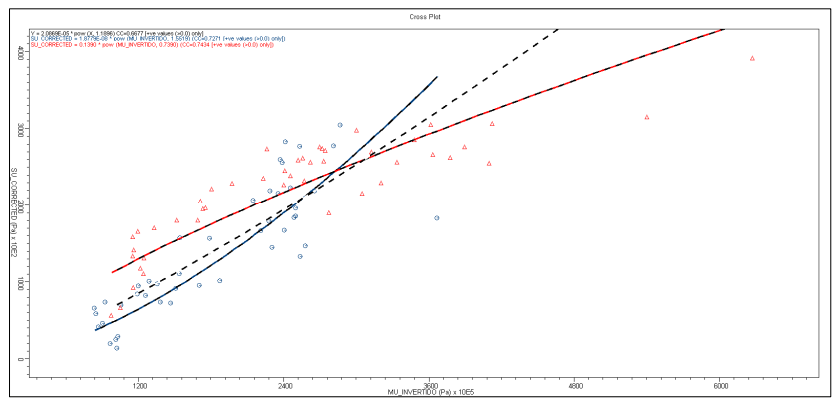

Figura 2 - Exemplo de ajuste do tipo "Power law" para a área de Albacora: ajuste individual (para o furo geotécnico GS-27 - em vermelho), ajuste individual (para o furo geotécnico GS-08 - em azul) e ajuste "global", (considerando ambos os furos - em tracejado), com base no crossplote entre Su (eixo vertical) - expresso em Pa X $10^{2}$ - e o módulo de cisalhamento invertido (eixo horizontal) expresso em $\mathrm{Pa} X 10^{6}$.

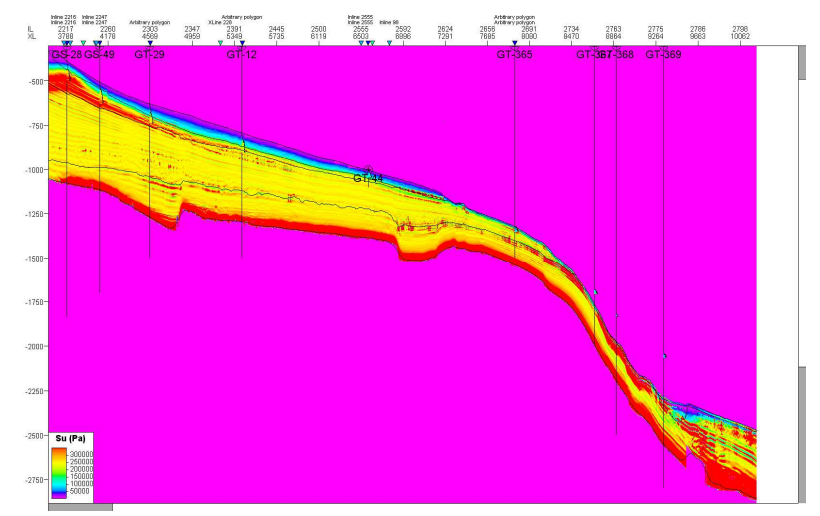

Figura 3 - Exemplo de seção de Pseudo-Su derivado do módulo de cisalhamento, com perfis de Su de furos geotécnicos GS e GT superpostos, para fins de comparação; eixo vertical em profundidade $(m)$ e extensão horizontal da seção de cerca de $20 \mathrm{~km}$; valores de pseudo-Su variam de 0 (magenta) a $3 \times 10^{5}$ $\mathrm{Pa}$ (vermelho). OBS: a cor magenta da parte basal da seção se deve à ausência de dados sísmicos de entrada. 


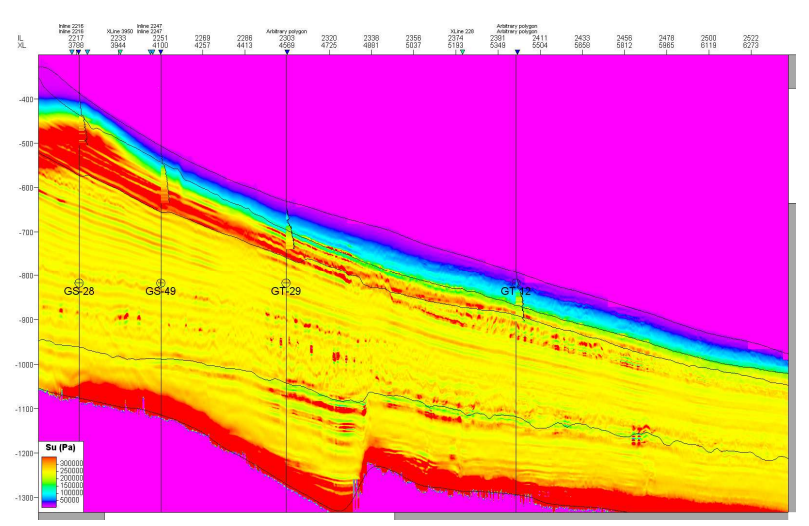

Figura 4 - Detalhe (porção proximal) de seção de Pseudo-Su derivado do módulo de cisalhamento apresentada na figura 3, com perfis de Su de furos geotécnicos GS e GT superpostos, para fins de comparação; eixo vertical em profundidade $(m)$ e extensão horizontal da seção de cerca de $5 \mathrm{~km}$; valores de pseudo-Su variam de 0 (magenta) a $3 \times 10^{5}$ $\mathrm{Pa}$ (vermelho). OBS: a cor magenta da parte basal da seção se deve à ausência de dados sísmicos de entrada.

\section{Vantagens e limitações da abordagem quantitativa}

Podem ser consideradas como vantagens da abordagem quantitativa aqui discutida:

a) a sua praticidade: com base no intensivo estudo realizado, esta foi considerada a forma de reproduzir da melhor maneira possível o comportamento geotécnico esperado (pelo menos em termos de variações relativas ou contrastes, o que, por si só, já pode trazer benefícios importantes às demandas de Engenharia) para as áreas investigadas. Pseudo-perfis geotécnicos de parâmetros de interesse como Su e qnet podem assim ser obtidos, sem a efetiva necessidade de novos furos geotécnicos.

b) Dentre as relações empíricas testadas, a opção por aquela que representa a "Power law" obedece a relações matemáticas que explicam fenômenos naturais da Física (por exemplo, as Leis de Kepler).

c) A resolução sísmica espacial vertical na seção rasa é a melhor possível, devido não somente às menores perdas cumulativas por atenuação, como também pelas menores velocidades sísmicas tipicamente associadas à mesma; isto é especialmente verdadeiro no caso das ondas cisalhantes, convertidas nas interfaces rasas ou mesmo no fundo do mar, a partir das ondas compressionais, e registradas em dados sísmicos multicomponentes; esta melhor resolução sísmica tende a implicar em uma correspondência superior com os dados diretos de furos geotécnicos, amostrados a intervalos centimétricos. d) Por fim, a extração de atributos com significado geotécnico a partir de dados sísmicos apresenta a vantagem de baixo custo de obtenção em relação às campanhas geotécnicas dedicadas (visto esses dados, geralmente bem amostrados em área e de boa qualidade inclusive, já estarem normalmente disponíveis, e prontos para uso, em todas as áreas onde haja interesse na caracterização geotécnica).

Por outro lado, aparentes limitações dessa mesma abordagem quantitativa (de certa forma até mais importantes de serem reconhecidas), incluiriam:

a) a abrangência antes local das relações matemáticas obtidas entre os diferentes parâmetros geotécnicos e módulos elásticos da Sísmica: pelo fato de as mesmas terem sido obtidas de maneira empírica (visto não ser conhecida uma relação analítica, genérica, entre aquelas grandezas), e com dados cativos às respectivas áreas investigadas, a validade das equações assim obtidas não deve ser generalizada. Outras áreas necessitariam de um estudo específico (embora seguindo a mesma metodologia desenvolvida) para obter relações matemáticas próprias, ou, mais especificamente, calibrar os coeficientes "a" e "b" para cada uma delas.

b) O comportamento anômalo esperado para alguns tipos de sedimentos, especialmente aqueles de origem nãosiliciclástica. Como exemplo, na área de Marlim foram observadas relações inversamente proporcionais entre Su e $\mu$ (ao contrário do que foi observado em termos gerais ao longo do estudo) no caso de algumas feições coralíneas superficiais, de arcabouço rígido, porém muito poroso, e assim, de baixa Vs (e baixo $\mu$ ). Também foi observado na mesma área de Marlim que sedimentos siliciclásticos com estrutura interna "desorganizada" ou caótica podem igualmente apresentar comportamento que difere daqueles sedimentos mais "bem comportados" ou organizados. Por fim, o trend exibido pelas curvas empiricamente obtidas reflete, essencialmente, a influência de sedimentos de granulometria fina (siltoargilosos), coesivos, os quais tendem a predominar na seção rasa das áreas investigadas.

c) A metodologia aqui desenvolvida prescinde da utilização de perfis de Vs (ou pelo menos de Vp, donde pudesse ser derivada a informação de Vs) e também de medidas diretas de densidade, ambos obtidos in situ, para fins de calibração do processo de inversão sísmica. No entanto, perfis deste tipo (em especial de Vs, chamados comumente de "cone sísmico") são, na prática, escassos na Margem Continental Brasileira. Como alternativa, a Vs poderia ser obtida a partir de Vp, nos casos em que este é disponibilizado a partir de outras fontes, como seria o caso de medidas de MSCL (multi sensor core logger), que muitas vezes complementam a amostragem geotécnico-geológica.

d) O intervalo de amostragem (vertical) típico da Sísmica, geralmente não mais refinado do que $2 \mathrm{~ms}$, resulta em um número não elevado de amostras ao longo do traço sísmico, no mesmo intervalo em profundidade coberto por um furo geotécnico co-localizado (que raramente 
ultrapassa 100-150 m), o que tende a reduzir o número de pontos reais de observação para correlação.

A aplicação da metodologia desenvolvida deve, de maneira consciente, reconhecer as limitações acima.

\section{Conclusões}

A metodologia desenvolvida para a extração de parâmetros geotécnicos a partir de dados sísmicos tem trazido resultados razoavelmente satisfatórios nas áreas onde foi aplicada, como atestado por comparações com dados geotécnicos reais não utilizados previamente para calibração. No entanto, limitações existem, as quais devem ser conhecidas de antemão, para uma mais criteriosa aplicação da ferramenta. Estas limitações são aqui discutidas.

\section{Agradecimentos}

Os autores gostariam de agradecer à PETROBRAS pela permissão de publicar este trabalho, bem como aos inúmeros colegas, tanto da PETROBRAS quanto da Schlumberger, que de alguma forma contribuíram com proveitosas discussões para o mesmo.

\section{Referências}

Sobreira, J. F. F., Lipski, M., Carvalho, L. A., Márquez, E., 2009. Simultaneous seismic inversion applied to geotechnical analysis of near seabed sediments. $11^{\text {st }}$ International Congress of the Brazilian Geophysical Society, Salvador.

Sobreira, J. F. F., Lipski, M., Carvalho, L. A., Marquez, E., 2010a. Geotechnical characterization based on seismic data approaches applied in Campos Basin, Southeastern Brazilian Margin. The Leading Edge, vol. 29, n. 7, p. 842846.

Sobreira, J. F. F., Carvalho, L. A., Lipski, M., 2010b. A more robust approach for shallow geotechnical characterization utilizing multicomponent pre-stack seismic inversion. Rio Oil \& Gas Expo and Conference, Rio de Janeiro.

Sobreira, J. F. F., Lipski, M., Pimentel, A. L., Carvalho, L. A., Artola, F. A. V., Márquez, E., 2010c. Caracterização geotécnica de solos marinhos empregando atributos derivados da sísmica: a importância da incorporação da informação elástica. IV Simpósio Brasileiro de Geofísica, Brasília.

Sobreira, J. F. F., Lipski, M., Carvalho, L. A., Artola, F. A. V., 2011. Extraction of geotechnical attributes from seismic data: towards a quantitative approach. XII International Congress of the Brazilian Geophysical Society, Salvador. 